\title{
Using the multiple scenario approach for envisioning plausible futures in long-term planning and management of the urban water pipe systems
}

\author{
Stian Bruaset ${ }^{*}$ (D) and Sveinung Sægrov
}

\begin{abstract}
Water utilities are facing the challenging problem of planning rehabilitation and renewal of their urban water systems for an uncertain future, which will be affected by climate change, demographic changes and extensive changes in the way the public perceives the water services. For the purpose of envisioning the future, the multiple scenario approach is presented, and its benefits and drawbacks are discussed in relation to other futures forecasting methods. The paper guides the reader on how to build scenarios that represent plausible futures for renewal planning of urban water and wastewater networks. For this purpose, a table is produced that gives an overview of relevant scenarios and their potential consequences. In the end, a case study from a Norwegian perspective is presented that gives the reader an overview of the process of building scenarios based on both qualitative and quantitative approaches.
\end{abstract}

Keywords: Scenarios, Urban water, Asset management, Sustainable development

\section{Introduction}

There are a number of publications and ongoing research within the academic community of urban water systems that focuses on future studies, sustainable development and the transition to Sustainable Urban Water Management (SUWM) principles. A lot of the work focuses on current and future challenges for the water sector, the need to apply sustainable solutions and the need for water utilities to implement SUWM principles through decentralization of the urban water system (UWS). A thorough and critical review of the SUWM principles are presented in Marlow et al. [1], which concludes that the water sector moves towards implementation of SUWM principles through a system hybridization process where new decentralized systems are sporadically built together with rehabilitation of the so called legacy systems. One of the key elements of this type of research is that the world is changing in terms of climatic, economic, technologic and socio-economic

\footnotetext{
*Correspondence: stian.bruaset@ntnu.no

Department of Hydraulic and Environmental Engineering, Norwegian University of Science and Technology, S. P. Andersens veg 5, Trondheim, Norway
}

parameters. SUWM proponents claim that traditional urban water systems are maladapted to the emerging challenges associated with these topics. The research works to mitigate the impact of these changes on the UWS, and at the same time tries to move the development of the UWS into the proper sustainable path. Studies made in order to envision future development of the changes within these areas and disciplines facilitates the water community in its endeavour into building sustainable water systems of the future.

This paper is addressed at the management people working with strategic level decision making in water utilities worldwide. The intention of the paper is to equip such managers in the academic field of foresight through the multiple scenario planning approach adapted to urban water pipe systems. The method described will facilitate managers in implementing a most robust and sustainable intervention strategy, by considering the uncertainty and the unpredictability of the future, both through aspects that are external to the urban water system and internal aspects. There will be some challenges related to these internal and external aspects. Key challenges when managing the urban water systems 
and planning for the future is the uncertain development of climate change, population growth, economy, regulatory frameworks, social conditions and technology. These are referred to as external factors and are the 'driving forces' [2] for future change. By contemplating the development of these factors in the future, it is possible to foresee and envision how the future might look like. Van Asselt [3] refers to this type of exercises as 'foresight' which, on a broad scale, is an attempt to make sense of the foreseeable future. Rhisiart et al. [4] calls these exercises for 'strategic foresight activities'. In the short (1-5 years) to medium term (5-10 years), the development of such factors can more or less be forecast, while in the long term (10-50 years) a multiple scenario analysis is recommended to allow for the increase in uncertainty [2]. Postma and Liebl [2] divides the driving forces into constant, predetermined (e.g. demographic changes) and uncertain factors (e.g. climate change). According to Waage [5] and Galloway [6], traditional long-term planning has looked at the development of these factors into the future as a 'cylinder of uncertainty', illustrating an expectation that variables have and will 'remain relatively constant over extended periods of time' [6]. The variability within the cylinder 'is limited to a snapshot of what has happened in the past' [5]. This traditional approach mostly uses the forecasting technique of extrapolating data and is based on a stable worldview [7]. The weakness of this prediction approach is the assumption that the past can describe the future. At long time horizons, the evolution of the system can even 'change the structure of the system itself' [8], making the use of present and historical data as foundation for future analysis even less suitable. This traditional approach of forecasting the future has failed to predict 'credible forecasts in the past few decades' [7], has failed to satisfy the forecasting needs of end users [7] and will fail to meet the challenges of non-stationarity of climate change and other uncertain factors into the future $[6,9]$. A response to the failures of traditional forecasting methods has been the rise of the multiple strategies and scenarios analysis. This approach, which is 'especially effective in dealing with uncertainties', has been applied in varying degree since the 1970s [2], but have gained momentum in the water sector for the past decade.

The water sector must come head to head with the fact that stationarity of nature no longer will be the case. Stationarity, which is 'the idea that natural systems fluctuate within an unchanging envelope of variability' [10], is and has been a fundamental concept within the urban water management. Brown and Farrelly [11] states that for the water sector to transition into the concept of SUWM, it will have to modify its traditional and linear approach to management into 'an adaptive, participatory and integrated approach'. This paper facilitates water utilities in transitioning into a path of sustainable development by presenting a procedure to envision and foresee different possible futures that utilities will have to plan for. In this endeavour, scenario building will help water utilities to shape a sustainable policy for their capital maintenance planning by projecting and visioning the possible challenges that utilities will have to face, and by embracing issues concerning uncertainty.

The purpose of this paper is to give a short introduction on the topic of scenario building and relate that to long-term management of the urban water pipe networks. Based on this limited review, the further objective is to establish a procedure for building scenarios which will facilitate water utilities in long- term sustainable management, where the focus is on rehabilitation and capital maintenance planning of the pipe networks. A Norwegian case study is then presented as an example of how the multiple scenario planning approach can initially be engaged from a broad perspective, then to be completed in smaller more specific sub case studies focusing on both quantitative and qualitative analyses.

\section{Establishing the context}

Marlow et al. [12] states that deterioration of the urban water assets is in varying degree 'common to the water sectors of many countries', which has come about due to a focus on short-term management in utilities. Utility budget restrictions and new emerging threats to the urban water and wastewater systems, like climate change and population growth, further complicate the sustainable management of assets. The first step to move away from the short-term focus into a more holistic approach where long-term effects and long-term solutions are evaluated is to establish the context of the future analysis by defining the boundaries of the system under investigation $[13,14]$. The system under investigation is the internal system, which in this case is the pipes in the urban water and wastewater networks. Pumps, valves, reservoirs, sensors, etc., are not included since they may require an approach that would diverge from the one proposed for pipes. The external system is the rest of the world that the water utility has no control over, but which may impact the internal system. When planning for the future, a water utility has to consider the area of influence (the internal factors) and the area of no influence (the external factors). The internal factors are mostly found in the internal system, but some may also be found in the external system, like political decisions and regulations which potentially can be influenced by water utilities. Blackmore et al. [15] call these factors for 'controls'. External factors, which the water utility does not have any influence over, are mostly found in the external system, comprising everything outside of the urban water and wastewater pipe networks. In 
Blackmore et al. [15], the external factors are simply called 'factors'. Examples of controls include system design and operation, governance, human capability and planning and development. Examples of factors include climate, demographics, natural disasters, societal changes and domestic and international economic changes [15].

\section{A short introduction to scenario planning}

According to Hallegatte [16], the 'uncertainty in future climate change is so large that it makes traditional approaches to designing infrastructures...inadequate'. The existence of a range of climate change models predicting different futures impedes the ability to forecast longterm effects on infrastructures. Van't Klooster and van Asselt [17] state that, in addition to uncertainty, the future exhibits unpredictability since many future relationships follow a nonlinear future pattern. According to Hallegatte [16], a long-term modelling approach should therefore look at a range of possible future outcomes, specified as scenarios, and identify the most robust solution for all outcomes. The sum of the scenarios should represent the whole range of plausible uncertainty. The most robust intervention performs best against the sum of scenarios representing the range of plausible futures, and not just against the most probable scenario outcome [18]. The most robust intervention strategy is therefore also the most resilient and the most adaptive measure. Van't Klooster and van Asselt [17] present the methodology of scenario axes for ranking the uncertainty and impact of scenarios, thus being able to identify the most threatening scenarios. It is a procedure that can be used to weigh the importance of scenarios in terms of what ought to be considered for future planning, and also when modelling the performance of potential intervention strategies [19]. Lempert et al. [20] suggests that creating a range of representative scenarios will promote something called Robust Adaptive Planning (RAP), which implies the implementation of a robust and adaptive intervention. This approach is in line with the introduced SUWM principles. The proponents of SUWM solutions, which imply an emphasis on local and decentralized solutions to urban water management, argue that robust and adaptive solutions are necessary in order to meet future uncertainties such as climate change, population growth and increased urbanization. The building of scenarios helps the decision maker in identifying the most adaptive long-term intervention strategy, equipping the urban water systems to meet the challenges of the future.

The estimated residual service life of pipes and pipe cohorts often assumes a stationary development over time of the external impacting factors. The impacts of these factors have traditionally been extrapolated from the past into the future. Forecasting how they will change with time is more difficult for some factors (e.g. climate change) than for others (e.g. population change) due to the uncertainty of how they will develop in the long term [16]. A way to handle this uncertainty is the use of scenarios, which embrace the idea of uncertainty instead of working to reduce it. The uncertainty is included within its multiple projections of the future. Scenarios try, in a broad sense, to answer the question 'what can happen?'. Bishop et al. [21] calls the development of scenarios 'the heart of futures studies', trying to tell different stories for multiple futures. Ashley and Tait [13] call scenarios visions of the future, emphasizing that scenarios are an envisaging of 'plausible and logically consistent versions of futures', also calling it a 'foresighting' process. A shift from the traditional forecasting method to the multiple scenario analysis revolves around a shift from reducing uncertainties to including uncertainties, by presenting a range of fundamentally different possible futures [7]. These future scenarios should describe generically different futures rather than variations on one theme [22], and according to van der Zouwen et al. [14], they should be 'fundamentally different from each other'. Waage [5] and Galloway [6] present 'the cylinder of uncertainty' as the old paradigm and the 'cone of uncertainty' as the new paradigm within long-term planning. The new paradigm planning approach, which is an illustration of the multiple scenario analysis described in Schoemaker [22] and in Bood and Postma [7], is also referred to as 'conical' planning. Miller and Waller [19] state that 'scenario planning is a qualitative method' that 'create coherent stories about possible futures'. We do however believe that the scenario planning approach opens up for using quantitative methods to estimate certain aspects of the scenarios, e.g. future population growth. Miller and Waller [19] go on to describe scenarios as a 'structured story creation' based on 'factual data,' human insight', intuition and creativity.

Investments in water infrastructures are long-term commitments that have consequences over the next 50 to 200 years. These long-term investments should be able to cope with a radically different climate in 100 years from now [16] and should be in line with future thinking within the academic and professional community. Longterm planning deals with a high degree of uncertainty, often linearly increasing with time as illustrated by the 'cone of uncertainty' in Waage [5] and Voros [23], and the 'funnel into the future' in Herz [24]. Both of these descriptions involve a 'window of opportunities' [24] in the future, which illustrate 'that the domain of uncertainty is expanding with time' [6]. Studies show that climate models forecast a wide range of different outcomes when looking at the same climate data $[5,16]$, emphasizing the uncertainty related to future modelling. The best way to treat this symptom of variability is to apply 
multiple outcome techniques to prepare for a range of plausible futures [5]. Scenario planning is stated to be the 'most prominent method for exploring the uncertainty of the future' [24] and is often used in projects dealing with long time frames and high uncertainties [14]. By designing a range of three, five or more scenarios, where two of them constitute the opposites of 'worst case' and 'best case' scenarios, the total uncertainty is included within the range of the 'forecasts'. The designing of a limited number of scenarios leads to a broad description of the future possibilities, and in doing so, 'scenario planning attempts to capture the richness and range of possibilities' of the future [22]. Building less than three scenarios could however distort and misrepresent the future as the combinations of scenarios would not cover the necessary future possibilities. The necessary number of scenarios should therefore be carefully evaluated in each case.

The multiple scenario approach also 'facilitates communication and negotiation between managers with deviating perspectives', and 'attack routines and habits' [7] within utilities. Thus, it becomes a tool to renew thinking and bring the management together. It is also a tool that can help managers overcome biases and help strategists to improve the overall quality of their decisions' [25]. Duinker and Greig [26] state that scenario analysis has become an integrated part of organizational pursuit of sustainable development. Companies and organizations have long been using scenario planning for strategic purposes, to 'explore uncertainties' and to review how current trends are shaping the future [6]. Another benefit of scenario planning is that it simplifies 'the avalanche of data into a limited number of possible states' [22]. The simplification procedure of the scenario planning helps to systematize an enormous amount of data into plausible imagined futures. For a detailed description on how to develop and construct scenarios, see Schoemaker [22]. Varum and Melo [27] review scenario planning literature for the last decade to give an overview of the publications on the topic, while Amer et al. [28] reviews the concept of scenario planning.

\section{Building scenarios}

\section{Building blocks}

When establishing the context of an analysis by defining the boundaries of the system under investigation, the external system is defined. This is called the relevant context [14]. The relevant context can include climate change, demographics, politics, economics and technological development. The relevant context and how the factors may develop into the future in the medium to long term is the basis for building scenarios. In order to build these scenarios, we need 'building blocks'. In explorative scenario building, which is described in
Börjeson et al. [29], the scenarios are based on external factors which are beyond the control of the water utility. Such factors, as presented in Table 1, represent the building blocks for the scenarios. Herz [24] calls them key factors. They can also be described as key drivers since they are the driving forces [2] for future change. In this paper, only factors that (a) can affect the physical condition of the pipes, namely deterioration, or which (b) can affect the need to improve network condition, are included. The factors are divided into direct (physically affecting network condition) and indirect (affecting the need to improve network performance, or can indirectly affect the physical network condition) impacts. The reason for including the given factors are based mainly on work within central European research projects, like PREPARED (see http://www.prepared-fp7. $\mathrm{eu} /$ ) and TRUST (see http://www.trust-i.net/). An evaluation on the balance and trade-off between details in the scenario planning and uncertainty and benefits is the reason for the selected number of specific external factors chosen for building blocks.

\section{Main and combined scenarios}

Scenarios are built by using one or several of the building blocks. The authors specifically distinguish between main scenarios and combined scenarios. Before the combined scenarios are built, the main scenarios, and variations of them, are identified (see Table 2). These scenarios are tailored for use within urban pipe systems rehabilitation planning. Variations of the main scenarios are described through their expected consequence impact, as defined in column two of the table. The consequence impact states whether the impact is negative or positive with regard to deterioration of pipes and the expected relative size of the impact. For each main scenario, there are scenario specifics described in column three. The scenario specifics describe expected future developments within the main scenario for both wastewater and drinking water pipe networks. The potential

Table 1 Building blocks (external factors) for building future scenarios relevant for the urban water and wastewater pipe systems

\begin{tabular}{|c|c|c|}
\hline & \multicolumn{2}{|l|}{ Type of impact } \\
\hline & $\begin{array}{l}\text { Direct } \\
\text { (physical) impact }\end{array}$ & Indirect (nonphysical) impact \\
\hline \multirow[t]{6}{*}{ External factor } & Climate change & Governing and regulating aspects \\
\hline & Demographics & Service level delivered to end users \\
\hline & & $\begin{array}{l}\text { Technologic and methodical } \\
\text { development }\end{array}$ \\
\hline & & Ambition of the water utilities \\
\hline & & Economy \\
\hline & & Organizational capacity \\
\hline
\end{tabular}




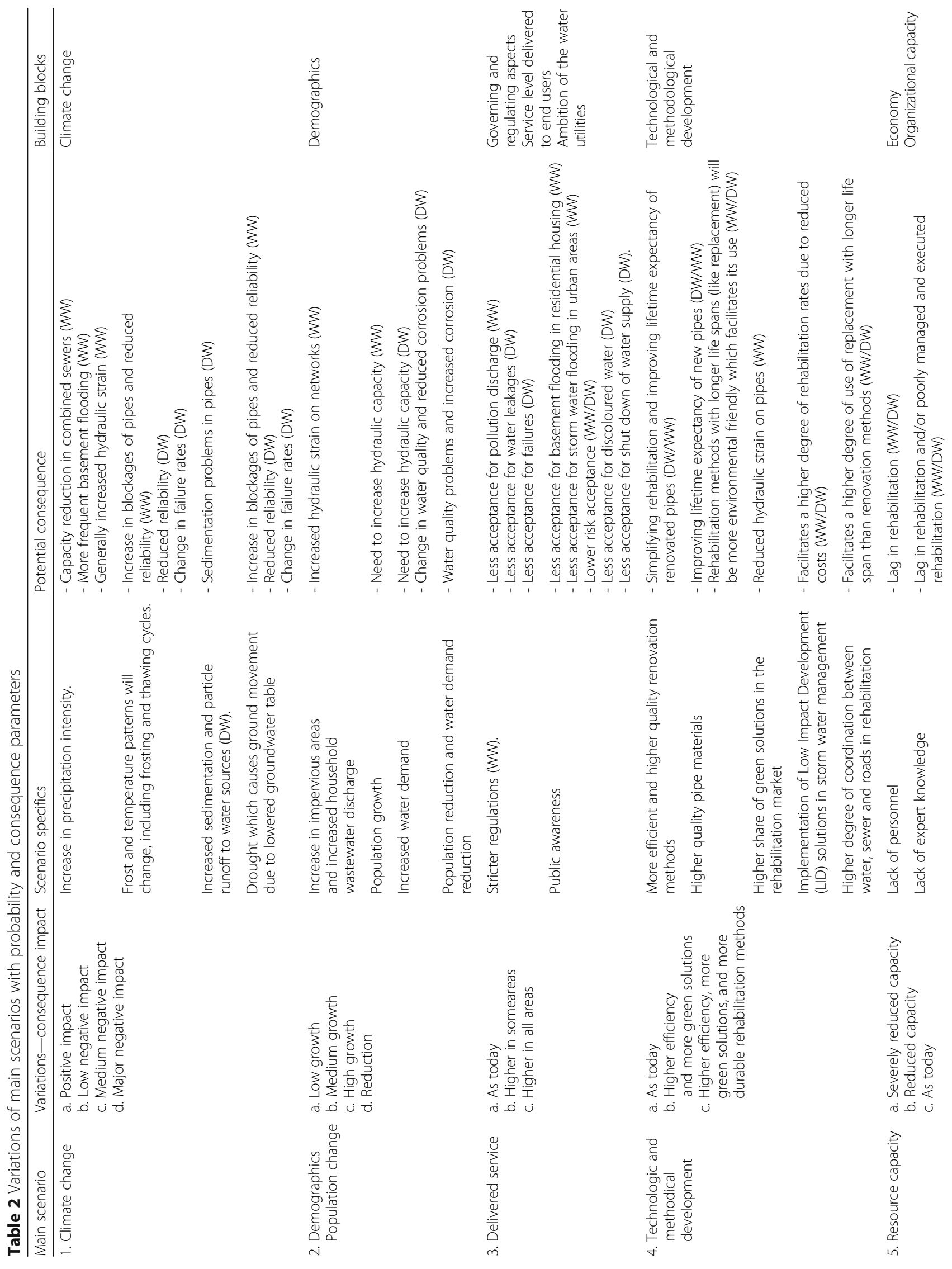




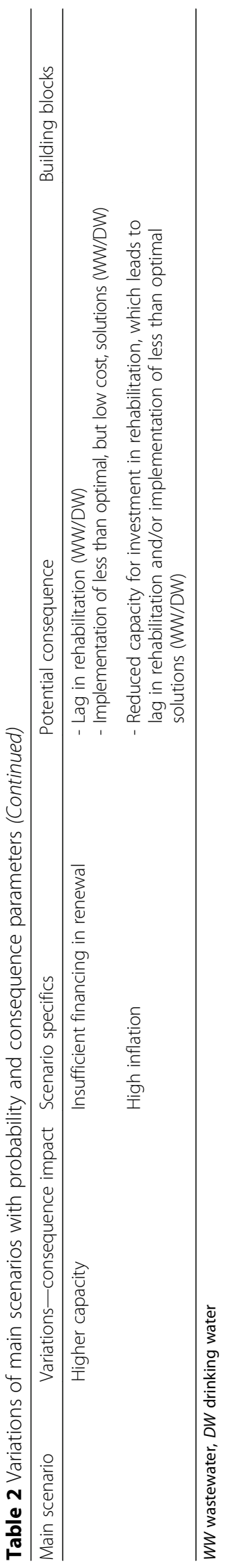


consequences of each of the scenario specifics are given in column four. When assessing the risk for a given scenario, it is the probability of the scenario specifics (column three) and the potential consequences (column four) that are used in defining the risks.

Sedlak [30] considers climate change to be the most important driver for long-term impacts on the urban water system. Whether this is a fact or not depends on which region of the world the urban water system is part of. Heavy increase in precipitation or heavy increase in the number and length of droughts could impact the pipe systems severely. Changes in average temperature and in temperature patterns could impact the reliability of the networks through changed ground movement and freezing and thawing cycles. Many utilities would however claim that urbanization will be more important for future impact on the systems, or that deterioration and lag in renewal is the main challenge. Lag in renewal is primarily caused by a lack in investments. Lack in investments is caused by a lack of available resources (personnel, organizational capacity and/or knowledge) and/or lack of economical means. A potential future change in available resources or economic means will further affect the efforts given to rehabilitation and renewal, which can be either positive or negative developments. Economical important factors are inflation and discount rates. These factors do not affect deterioration per se, but can affect rehabilitation efforts and thus could affect deterioration in the long term. An estimation of future inflation or the consumer price index based on centralized governmental appreciations of the value of money could be used to estimate the future economical capacity of a utility.

Many of the cities worldwide will experience further population growth in the coming decades [31, 32]. This creates a huge challenge for the urban water systems and is therefore considered as a main scenario. 'Delivered service' and 'technologic development' are also considered as main scenarios since they both are expected to be continually improved in a state of the art society. Moving towards sustainability, it is not reasonable to avoid an improvement in both rehabilitation efficiency through technological evolution and service levels, or at least to maintain the current levels.

The combined scenarios are constructed by merging variations of the main scenarios, as given in columns one and two of Table 2. Combined scenarios therefore give more possibilities of describing the potential futures. A best case and worst case scenario can be constructed from the variations of the main scenarios by accumulating the most positive and most negative impacts, respectively. In between the two extreme scenarios, there should be one or more scenarios to fill the void, where one scenario could possibly constitute the most probable one.
Table 2 is the foundation for water utilities to build their own scenarios, and works as a 'buffet' where utilities can combine variations of main scenarios as they see fit for their local situation.

\section{Using scenarios}

Scenarios can be used, applied and implemented in a range of ways. The most basic use of scenarios is just to improve the understanding of the future. Scenarios can be used to stimulate thought and give the user some understanding of the uncertainty of the future by giving some 'window of opportunities' [33] and by presenting a range of fundamentally different futures that can come true. The next level of using scenarios is to model the possible effects of the future on the system under investigation. Scenarios can for example be used to understand the impact of the future on urban water pipe systems. This enables utility managers to understand not only the future but also how the system might react in response to external changes happening in the future. Knowledge about this gives the manager some time to prepare and plan to implement changes that are robust enough to cope with the changes. The third level of using scenarios is to assess a range of future intervention measures against the range of future scenarios, which enables utility managers to choose the most robust and sustainable long-term solution. A number of indicators can be used to measure the sustainability performance of the intervention measures against the range of scenarios. Rehabilitation interventions can in this case be represented by different rehabilitation strategies.

\section{Risk management of scenarios}

One of the objectives of multiple scenario analysis is to explore risks [26]. The main objective of the risk management is to identify the conspicuous risks in the long term that are important to minimize, and assess the best available risk treatment. Each scenario is representing an undesirable event, because it potentially unleashes a number of consequences for the urban water systems. The placement of the scenarios within a risk matrix is basis to score the risks based on the level of risk severity, which later can be used when evaluating different future intervention strategies against the range of scenarios.

\section{Assessing the probabilities and consequences of scenarios}

The future probabilities and consequences of the scenarios can be assessed with quantitative or qualitative methods. The approach taken should be based on available data and resources in the utility, and on available data for the future development of the scenarios. It is always a challenge to quantify the long-term uncertainty of external factors, both because of the long time frame and because of the intricate system of parameters that 
can influence a certain factor. The goal of an uncertainty assessment would not be to quantify an exact uncertainty value, but to evaluate the range of the uncertainty and be able to place the risk of the scenario within a risk matrix (see Fig. 1). For a qualitative approach, a project group can for example be established with persons of different scientific backgrounds. This would facilitate a broad discussion on the possible consequences of different scenario impacts. A process called the Delphi process is often applied in such groups, which is a group iterative process that is based on structured communication. The group contestants answer questionnaires in several rounds, each time revising their answer based on the input from the group. The answers from the contestants are kept anonymous. It is an iterative process in that they take several rounds to revise their answers until they converge towards some common understanding and a final answer. A predefined criterion is sometimes used to define when the process is finished. Such a criterion could be the number of iterative rounds or the degree of convergence. The same process could be used for defining the probability of the given scenarios if no statistical data is available.

The consequences of scenarios are best defined with the above described process, with the assumption that the project group consists of a diverse range of experts that have different scientific views and approaches. The probability of scenarios is however best described by a quantitative approach, as long as data is available. Operational data from the daily operation of the urban water networks can for example be used in a statistical analysis as basis for asserting some conclusions about future developments, and thus be able to produce some uncertainty.

\section{Risk assessment and evaluation}

The risk analysis involves a quantitative or qualitative assessment of the probability of the risk happening and the consequence if the risk should happen. This is in line with the definition of risk [34, 35]. Risk probabilities and consequences should be divided into levels representing severity of a potential event. The probability level represents the probability for the event (scenario) to occur within the prognosis period, and the consequence level represents the relative size of the positive or negative impact on pipe condition and performance. Ugarelli and Røstum [36] and Ugarelli et al. [37] show approaches on how to scale probabilities and consequences into severity levels, which can be quantitative or qualitative. Ugarelli and Røstum [36] define consequence scales for different dimensions. The dimension of pipe condition and performance is the consequence scale considered as relevant for this paper.

An illustrative example of a risk analysis and evaluation of future population growth is shown in Fig. 1. The probabilities and consequences are basis to place the risks within the risk matrix. The risk severity in the example is represented by three levels: high, medium and low. These three levels are illustrated with different shades of colour. By including five or more levels, it is possible to make a clearer distinction between scenarios. To score the risk level severity, we suggest that high risks (dark colour) are given a score of three, medium risks (light colour) a score of two and low risks (no colour) a score of one. The risk severity score is used to weigh the importance and severity of the scenarios when assessing possible future outcomes.

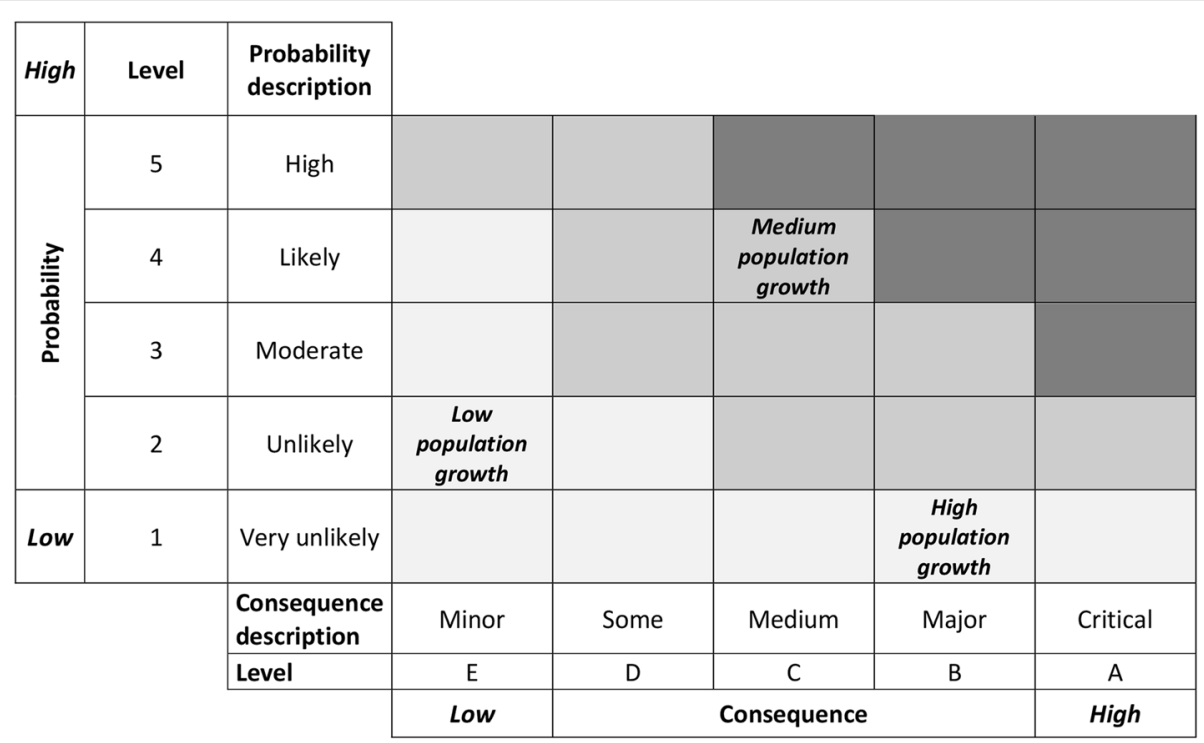

Fig. 1 Example of consequences and probabilities of future population growth scenarios used in risk analysis and risk evaluation 
A case study of the Norwegian drinking water pipes Many of the municipalities in Norway are experiencing lag in renewal of their water and wastewater pipes and are at the same time experiencing effects of climate change like increased intensity of rainfall events. It is expected that the future will bring more rain, heavier rainfall intensity events and a change in atmospheric temperature [38], which might impact the operation, maintenance and rehabilitation of the urban water systems [39]. The length of the Norwegian drinking water distribution system is around $46,000 \mathrm{~km}$, with more than $1 / 3$ of it being constructed before the 1970s. Pipes between 1940 and 1980 have varying quality due to challenges caused by poor construction practices and poor construction standards of the pipes. Pipes laid the first 20 years after the second world war are the most troubled pipes. The network consists mostly of cast iron, ductile iron, and plastic pipes (PE and PVC). An initiative by the Norwegian interest group for water utilities, 'Norsk Vann' ('Norwegian Water'), and ten of the largest municipalities in Norway, looks to improve modelling for long-term rehabilitation and investment needs in order to better predict the future investment needs. A project has been running for 3 years with the objective to improve the modelling approach and to calculate the national future investment needs of the Norwegian water and wastewater pipes until 2070. As part of this, the scenario approach was applied in order to review the longterm impacts of external factors on the pipe networks. This paper presents the primary initiative to produce future scenarios for the drinking water pipe network.

The project was handled by a core research group consisting of two researchers (the authors) and two managers from different municipalities, and a project group consisting of representatives from all the municipalities, the interest group Norsk Vann, and the two researchers. The initial work consisted of a questionnaire sent to four of the municipalities and a different questionnaire sent to researchers within the social sciences discipline. In the questionnaire, the municipalities were supposed to point to, according to the experience of the utility itself, the most challenging problems facing the water pipe systems in the future. The social science researchers were asked about topics of risk and service provision related to society and the future. All of the four municipalities named ageing and deterioration of networks as one of their main challenges facing the future. This indicates a lag in renewal. Population growth was indicated as a challenge by two of the municipalities, while one municipality also pointed to future climate change as a problem.

Based on the feedback from municipalities within the questionnaires, work with the project group, and a literature study, the core research group produced a document to identify the most relevant future consequences for the
Norwegian urban drinking water network based on the main scenarios identified in Table 2. A description of the problems and possible solutions as to how they can be analysed into the future was discussed. A summary of this is given in Table 3. The scenario specifics and consequences that are not included were omitted from the analysis based on the screening process (interviews, project group and core research group discussion). The omitted scenario specifics were just not considered as relevant for Norwegian conditions. It was also decided to focus on what seems to be the most challenging future trends for the Norwegian water sector. What remained was a list of relevant problems to be addressed more closely. These are listed in Table 3.

The results from the preliminary round of screening led to the selection of the scenario specifics as defined in Table 3, representing the most pressing concerns of future consequences. The more thorough assessment depicted in this table resulted in more specific conclusions for each scenario specifics and their relevant analysing approaches. This led to a branching out of the case study into smaller sub case studies focusing on specific parts. The conclusions for the analysing approaches were the following:

- Analysing approaches 2b, 3c and 3d: it was concluded that these problems were better solved by O\&M procedures instead of solving them with optimized rehabilitation levels. There was therefore produced no follow-up tasks to these approaches. This was substantiated by cost- benefit and sustainability reasoning, as other more suitable solutions than rehabilitation are available at lower costs and with less impact.

- Analysing approaches 3a, 4a and 4b: A qualitative case study based on interviews was considered to be the best solution for these approaches. Relevant people for each approach were contacted for an interview. The selection of relevant people was based on their relevance for the subject matter, their experience and their up-to-date knowledge.

- Analysing approaches 1a, 1b, 1c, 2a, 5a, 5b and 5c: A quantitative case study based on data analysis was considered to be the best solution for these approaches. We found it necessary to create three dedicated sub case studies to cover all the scenario specifics. These sub case studies are explained more in detail below.

- Analysing approach 3b: We found that there is little Norwegian specific data available on the public perception of risk levels related to water and wastewater systems. The topic is intricate and comprehensive, and any conclusion should only follow a project that has done studies into a representative number of people. New knowledge on 
Table 3 Relevant future scenarios, potential consequences and analysing approaches identified in the case study

\begin{tabular}{|c|c|c|c|}
\hline Main scenario & $\begin{array}{l}\text { Most relevant scenario } \\
\text { specifics }\end{array}$ & $\begin{array}{l}\text { Water distribution potential } \\
\text { consequences }\end{array}$ & Problem description \\
\hline Climate change & $\begin{array}{l}\text { Frost and temperature } \\
\text { patterns will change, } \\
\text { including frosting and } \\
\text { thawing cycles }\end{array}$ & $\begin{array}{l}\text { - Reduced reliability } \\
\text { - Change in failure rates }\end{array}$ & $\begin{array}{l}\text { Reliability and failure rates can } \\
\text { be impacted by temperature } \\
\text { changes which impacts ground } \\
\text { movement and frosting and } \\
\text { thawing cycles. }\end{array}$ \\
\hline $\begin{array}{l}\text { Demographics } \\
\text { Population } \\
\text { change }\end{array}$ & Increased water demand & $\begin{array}{l}\text { - Need to increase } \\
\text { hydraulic capacity } \\
\text { - Change in water quality } \\
\text { and reduced corrosion } \\
\text { problems }\end{array}$ & $\begin{array}{l}\text { Population growth leads to } \\
\text { increased water needs. Will this } \\
\text { lead to a need to upgrade the } \\
\text { hydraulic capabilities of the water } \\
\text { supply? Increased water demand } \\
\text { might actually improve water } \\
\text { quality with regards to corrosion } \\
\text { problems. }\end{array}$ \\
\hline
\end{tabular}

Population reduction and - Water quality problems water demand reduction and increased corrosion

Delivered Public awareness - Lower risk acceptance

service $\begin{array}{ll}\text { Technologic } & \text { More efficient and higher } \\ \text { and methodical } & \text { quality renovation methods } \\ \text { development } & \end{array}$

Higher quality pipe materials

Resource capacity
Lack of personnel

- Lag in rehabilitation
Many Norwegian systems are already operating under low flow velocities due to high capacity on networks, so this will probably not impair the situation to any noticeable level.

Means a need to generally improve network condition and system performance. Risk should at the minimum be maintained.

- Less acceptance for discoloured water

- Less acceptance for shut down of water supply
Increased problems with corrosion, particles and sedimentation increase the probability of events leading to resuspension of particles in network and discolouration of the water.

A possible increase in failures on network will cause an increase in repairs. This, together with increased public demand to water supply, will increase the expectations and demands to continual supply of water.

Higher quality no-dig methods, both the materials and the procedures. Better and more effective installation procedures.

Material quality will improve in time with better raw materials and better production standards. Technical approval of pipes will probably be a standard for Norwegian water utilities.

Old network. Poor performance. Too little investment in renewal of pipes. We expect that there will be an increased rehabilitation need due to the demographic echo of past construction periods.
Suggested analysing approaches

(1a) Data analysis: correlation between failures and temperature

(1b) Data analysis: correlation between failures and frost/thaw temperature ranges

(1c) Look at future climate research literature

(2a) A case study of the Oslo water supply by looking at the impact of different water demands on hydraulic capacity

(2b) Estimation of de population from areas from the Norwegian national statistical database. These problems should however be solved with O\&M instead of increased renewal

(3a) Questionnaire to municipalities to review their perception of network risk levels

(3b) A review of the public perception of risk levels related to water and wastewater systems

(3c) Data analysis of discolouration data. However, these problems are best solved with O\&M techniques instead of rehabilitation (too costly)

(3d) Can be solved by techniques for repair and proactive maintenance. Increased renewal is probably not the best solution

(4a) What kind of technological advancements can be expected in the next 50,100 years? Interviews of Norwegian pipe producers

(4b) Interviews of Norwegian pipe producers on future technological advancements

(5a) Analysis of the Norwegian water network age and construction periods 
Table 3 Relevant future scenarios, potential consequences and analysing approaches identified in the case study (Continued)

\begin{tabular}{|c|c|c|c|c|}
\hline Main scenario & $\begin{array}{l}\text { Most relevant scenario } \\
\text { specifics }\end{array}$ & $\begin{array}{l}\text { Water distribution potential } \\
\text { consequences }\end{array}$ & Problem description & Suggested analysing approaches \\
\hline & Lack of expert knowledge & $\begin{array}{l}\text { - Poorly managed and } \\
\text { executed rehabilitation }\end{array}$ & $\begin{array}{l}\text { Too few students are being } \\
\text { educated for the water sector. } \\
\text { Poor management leads to poor } \\
\text { performance of the networks. }\end{array}$ & $\begin{array}{l}\text { (5b) Analysis of trends in educational } \\
\text { data compared to the future } \\
\text { need for the water utilities }\end{array}$ \\
\hline & $\begin{array}{l}\text { Insufficient financing in } \\
\text { renewal }\end{array}$ & $\begin{array}{l}\text { - Implementation of less } \\
\text { than optimal, but low } \\
\text { cost, solutions }\end{array}$ & $\begin{array}{l}\text { Implementation of less than } \\
\text { optimal solutions will lead to } \\
\text { poor long-term performance in } \\
\text { increased future investment need. }\end{array}$ & $\begin{array}{l}\text { (5c) Calculation of future investment } \\
\text { needs, which then should be } \\
\text { compared to currently planned } \\
\text { investments in rehabilitation }\end{array}$ \\
\hline
\end{tabular}

this topic will be pursued through the potential of a national project, which means that we will not be able to include this aspect into the scenario building.

The organization of the most pressing scenario specifics and their assessment led to the establishment of some central sub case studies, which will be topics for later more detailed publishing. Each of the case studies were designed to focus on consequences related to the specific main scenario and would look at probability where possible. Some of these sub case studies are already underway and results are available. It is planned that all of these case studies will be carried out within the next year, in time to support the national Norwegian project on investment needs in the Norwegian water and wastewater networks. In the following, the financial and resource use of the core research group and the project group are discussed, and the sub case studies and their expected results are explained in more detail.

\section{Financial and staff resource use}

During the study, the project group met only once a year for 2 years. The project groups' contribution to the project was therefore very limited, and restricted mostly to giving feedback to the overall process of the scenario planning. The annual project group meeting lasted for a day, but that meeting included discussions relating to several topics, and not just the scenario planning. The number of persons in the meetings was seven, where four of them had to travel from other parts of the country. This means that seven persons had to clear 1 day of work in terms of resource use, and four people had to order flights from different parts of the country in terms of financial resource use. No overnight accommodation was necessary. In addition, the main author of the paper used about 1 day to prepare for each of the meetings.

As the project group was mainly concerned with giving feedback, all the heavy lifting of the project was left to the core research group. The authors were tasked with the main bulk of the work, with the two managers from the municipalities assisting the authors whenever necessary, for example in the distribution of questionnaires, helping answering pressing questions, data gathering, etc. The managers' resource use did not exceed 1 day of work in addition to the annual project group meetings (which they attended). For the main author, the bulk of the work was the identification of relevant future scenarios, as given in Table 3, setting up the variations of sub case studies, and producing questionnaires for the interviews. It is estimated that the two authors used three to four weeks of combined work in total for producing Table 3 results and setting up all the sub case studies. This work included a lot of discussion between the authors.

As each of the sub case studies go into more details, it is expected that the resource use on some of them are higher than for the initial production of Table 3 and its follow-up. This is evidenced by the work already done in sub case study 4 through the work of Simonsen [40]. He used about 2 months in total for setting up the hydraulic modelling tool, establishing the scenario cases, importing and running the hydraulic model, and assessing, presenting and discussing results. Sub case study 3 has been an ongoing work parallel with the writing of this article, and it is expected that the total amount of time used on the data analysis in the case will amount to about 2 months. That includes data gathering, data screening, literature study, data analysis and assessment of results. Generally, it seems that quantitative analyses take more time than qualitative analyses. Simonsen [40] also contributed to sub case study 1 , which included less resource use than study 4 . The reason seems to be that quantitative analyses are based on more comprehensive data sets, which makes data gathering, data screening and data analysis more time consuming.

Outside of air travel for some of the project group members, the study did not lead to any direct costs and use of financial resources. The main contribution to resource use in the project was therefore the working hours spent by members of the two project teams.

\section{Qualitative case studies Sub case study 1}

This case study was established in order to support analysing approach $3 \mathrm{a}$ and the scenario of delivered service. As stated earlier, a questionnaire was sent to four large 
Norwegian water utilities. The questions revolved around their views concerning level of service and their ability to deliver satisfying service levels now and in the future, the increasing expectations from customers concerning level of service, population growth and its accompanying challenges, water supply reliability, customer willingness to pay, the future ambitions of the utility, and future challenging trends for the urban water systems. Another questionnaire was sent to two social researchers at the Norwegian University of Science and Technology. The interview questions and their results are found in Simonsen [40].

The results will be used to review topics of risk, service levels and reliability from the utilities' point of view, to assess if there is any indication among the water sector professionals that service levels will have to rise in the near future with the effect that rehabilitation efforts will have to be increased.

\section{Sub case study 2}

This case study was established in order to support analysing approaches $4 \mathrm{a}$ and $4 \mathrm{~b}$ and the scenario of technologic and methodical development. Four leading persons from different pipe producers in Norway were interviewed with questions revolving around the development of pipe quality, pipe material, research and future technologic advancement.

The interviewed people are central when it comes to the specific pipe materials, and the research work being put into the development of pipes. The results will be used to foresee future technological advancements in pipe materials and pipe quality in the next 50 years or so. This can then be used to say something about the quality of the pipe systems in the future, and if we can expect pipes to last longer than today.

\section{Quantitative case studies Sub case study 3}

This case study was established in order to support analysing approach $1 \mathrm{a}, 1 \mathrm{~b}$ and $1 \mathrm{c}$, and the scenario of climate change impact. Extensive amounts of climate data and operational data exist for the Norwegian pipe networks, which facilitates a wide and extensive data analysis. The situation is therefore tailored for a quantitative analysis. Long-term climate predictions for Norway until the year 2100 are prepared by the Norwegian Environmental Agency [38], where different emission scenarios are the basis to calculate ranges of expected air temperature, precipitation, sea level rise and other hydrological data. The first step of the case study is to look for correlations between operational data (e.g. failures on drinking water pipes) and climate data (e.g. temperature), and if any correlation is found, extrapolate that correlation into the future based on the different emission scenarios. The end result is a prediction of the effect of climate change on the reliability of the urban water systems. This approach is a combination of the standard prediction method and scenario planning, as the prediction is based on different future emission scenarios.

\section{Sub case study 4}

This case study was established in order to support analysing approach $2 \mathrm{a}$ and the scenario of population growth. The result of this case study is already available in Simonsen [40]. Part of the water network of one of the largest cities in Norway was chosen as it represents the situation in many of the Norwegian cities: high water pressure, varying topography and medium density in population. The case study looked at the impact of three factors on the hydraulic reliability of the water supply: population growth, leakages and deterioration of the network. Deterioration was modelled by increasing the roughness of the pipes. Single scenarios were run for these parameters, and the following four combined scenarios were tested:

- Increase in maintenance (to reduce leakages and roughness) and medium population growth

- Small increase in maintenance and medium population growth

- No increase in maintenance and medium population growth

- Decrease in maintenance and high population growth

The study found, despite the hydraulic oversized network, that the scenarios will lead to some pressure deficits in the network. The worst scenario will lead to a moderate impact on network pressure and will cause a need for an upgrade of the hydraulic capacity in order to maintain the reliability of the water supply system. Results show that a number of nodes (less than 10\%) will not maintain the necessary service pressure. These results will be used to assess the need for a hydraulic upgrade of the water supply system based on different scenarios.

\section{Sub case study 5}

This case study was established in order to support analysing approach $5 \mathrm{a}$ and $5 \mathrm{c}$, and the scenario of resource capacity. There already exists an analysis of trends in educational data compared to the future need for the water utilities. This analysis shows that there are too few people being educated for the Norwegian water sector, which might impact the future ability of water utilities to meet future challenges.

In connection with the national project on investment needs in the urban water sector, there will be an extensive data analysis on the composition of the water network, including the distribution of ages and 
construction periods. Based on these distributions, and on unit costs for rehabilitation, there will be a calculation of future investment need. These three approaches (educational data, lag in rehabilitation, investment need) will help the water utilities to understand the problems they are facing with regard to the composition of their networks, upcoming investment needs and future resource capacity.

\section{Discussion}

By including several people in the scenario building process, possibly from different branches of science, it helps to reduce subjectivity and helps to drive discussion within a utility. The combining of scenarios with risk management helps to strengthen the long-term planning by embracing uncertainty and emphasizing high-risk future scenarios. The selection of scenarios, their qualitative or quantitative determination and the risk analysis are vital to obtain a vision of the future which is representative and which reduces bias and the possibility of choosing a failed strategy when implementing an intervention. A failed strategy could result in extensive environmental impacts, larger than necessary costs and high social impacts, especially for future generations. As for now, there are a total of 5 main scenarios and 13 potential consequences specifically designed for building scenarios for the urban water networks. The variations of the main scenarios give the user variability to adapt scenarios which are suitable for the local situation.

Key external elements for the urban water systems are hard to predict or foresee into the future. Elements like climate change, population growth, economy, regulatory frameworks, social conditions and technology will impact the necessary flow of money and skilled labour into the urban water systems management. The big question is how to foresee the future and manage the systems in a way that makes water utilities prepared for different kinds of changes and different kinds of impacts. Past solutions like extrapolation of data from the past into the future has often proven lacking in predicting reliable futures, especially as we expect the non-stationarity of climate to be more present in the future. There will be an uncertain development of the external factors into the future, especially in the long term. We have argued that a multiple scenario approach is the best solution for handling these long-term uncertainties, by embracing uncertainty within its approach. The uncertainty is embraced by the building of multiple and fundamentally different futures. When the scenarios additionally are ranked with the risk approach, uncertainty is at the same time reduced by preparing for the most high-risk scenarios.

However, there are weaknesses and limits associated with scenarios. Scenario planning have evolved from an emphasis on quantitative methods to an emphasis on qualitative methods, and even though scenario planning is supported by an increasing amount of tools, it will remain 'a highly subjective art' [28]. The main weakness of the quantitative methods is that they mostly rely on historical data and an assumption that past and current trends will continue into the future. This is in contrast to the perception of the future in literature such as McCarl et al. [9] and Milly et al. [10]. Quantitative methods are therefore usually not recommended for projects with long time horizons [28]. The longer the time horizon, the more suitable is the qualitative approach for scenario building. Also, quantitative analyses are primarily focused on the analysis and forecast of a single parameter, sometimes without taking into consideration how variables and parameters may impact each other. On the other hand, the primary weakness of the qualitative method lies in its subjective nature. The inclusion of humans and the human mindset will automatically lead to subjective input. There are ways to counteract this (the Delphi process, wide variety of experts included in scenario building), and recent literature [25] shows that the scenario approach can help to reduce bias in strategic planning. The recommended number of scenarios in literature varies, but it is recommended to build between three and six scenarios [28]. Generating more than five might be too costly though and will in most cases not be justifiable [28]. On the other hand, less than three scenarios might be too few because they will probably represent two extreme situations (best and worst case). Everything in between will be undefined. Having three scenarios might cause an unhealthy focus on the middle scenario (which is also the most probable) [28]. Choosing the appropriate number of scenarios is therefore critical, is not an easy feat and will involve the risk of choosing too few or too many. For building each specific scenario, the literature recommends using between three and eight factors, specified as uncertainties [28]. Scenarios are built of these factors, as also discussed extensively in section 4. Using less than three factors can simplify the situation too much, and relying on more than eight factors will lead to high scenario production costs. Furthermore, failing to produce plausible, internally consistent and relevant scenarios will render them unfit for use in scenario planning, showing that there are major concerns in the process besides facts and number of scenarios and factors. The focus on details in the scenario planning process is therefore fundamentally important, and shows that scenario building is not easily achieved, nor should it be taken lightly, otherwise biasing can occur. Meissner and Wulf [25] show that only a complete application of the scenario planning process can ensure their so called 'debiasing' effect. Approaching scenario planning in a simplified or reduced manner will therefore not assure 
users of a non-biased decision-making process, and is on this basis naturally not recommended.

The Norwegian case study for scenario building shows how variations of main scenarios (Table 2) can be applied in a real case. The case study implements both the qualitative and the quantitative approach, and shows how both of these methods can complement each other to build future scenarios that cover a wide range of topics. When data is not available or cannot be fully trusted (e.g. when extrapolation is not suitable), a qualitative approach where people from different scientific areas participate is often the better alternative. By leaning on both of these approaches, it gives a utility the possibility for a wide range of inputs to the scenario building, which facilitates a broad approach to the problem. Problems can however arise when a utility needs to rely too much on data analysis or too much on the subjective input of persons. Data analysis often lacks the broader thinking of the qualitative approach, which specifically brings the social and technological aspects into play through the assessment of service provision and quality, risk perception, public awareness and technological development. On the other hand, the qualitative approach is for the most part subjective in that the knowledge input is given through humans. The Delphi approach or the gathering of information from a number of interviews is one way to reduce the subjectivity of the input. The best approach is therefore to merge knowledge from data analysis (quantitative) with knowledge gained from experts of different areas (qualitative). This approach is recommended in Amer et al. [28], which claims that these two approaches are 'complementary and strengthen each other when used together'. The case study shows on a broad scale how important it can be to use both of these approaches in a combination to describe scenarios. Relying only on the qualitative approach would exclude important impacts from population growth and climate change, while relying only on the quantitative approach would exclude important impacts concerning risk, service levels, reliability and technology, which includes the often-overlooked social aspect. Besides showing the importance of using both quantitative and qualitative approaches, the case study shows that all of the described sub case studies are relevant for describing the main scenarios. If one of the sub case studies is ignored, it can impact the representativeness of a scenario, and may render it non-relevant. The necessary width of the approach, and its appetite for details, is therefore the Achilles' heel of the method.

The multiple scenario approach can help utilities in reaching sustainability goals and/or enable them to implement suitable SUWM solutions and principles since it lays the groundwork for planning optimal intervention solutions. Without knowing what to plan for, it is hard to implement the right kind of decision. With the multiple scenario approach, utilities have the tool available to minimize the negative impact of an uncertain and unpredictable future. SUWM proponents claim that traditional urban water systems are maladapted to the emerging challenges of the future. One way to support this claim is to apply the multiple scenario analysis in order to visualize the future challenges that the urban water systems are facing, and see if the traditional legacy systems can meet these challenges in a sustainable way or not.

\section{Conclusions}

Based on a review of available literature on long-term planning principles within the urban water system and the theory of future studies, the authors have suggested a process based on multiple scenario planning to be the most suitable to plan long-term rehabilitation and investment needs in the urban water and wastewater pipe systems. In the last several decades, the multiple scenario approach has emerged as a central method to envision different futures in future science circles, and have gained momentum also in the water sector. This has come about due to the lack of other methods in predicting or foreseeing reliable futures. Also, the large variability and expected development in factors impacting future change, like climate change and population growth, has forced scientists into creating a tool that envisions different kinds of futures, instead of trying to predict that single, most probable future. Some of the advantages of scenario building are that it imagines a 'window' of future possibilities, it embraces uncertainty and it facilitates discussion among water utility engineers and managers.

The paper presents building blocks for scenarios, main scenarios, variations of main scenarios and combined scenarios as a process to understand the building of scenarios for the urban water systems. Table 2 represents the final product of this process, which gives the user a menu of which to build scenarios that is suited to local challenges and situations. This table describes each of the main scenarios in more specific terms and lists all potential consequences of significance.

The paper also incorporates the aspect of risk in the multiple scenario building. One of the objectives of multiple scenario analysis is to explore risks in the long term. This is considered an important part of the scenario building since long-term management of the urban water networks deals with many uncertainties, especially in a world dealing with non-stationarity, technological development and large population movements. The risk process is formed in a way to help water utilities in emphasizing scenarios that constitute a high risk to the future of the urban water systems, and de-emphasize scenarios that constitute a low risk. The scenarios are therefore ranked based on their level of risk severity. 
The case study gives the reader an impression of how the scenario table (Table 2) can be used to build scenarios that fit the local situation, and gives suggestions on how specific scenarios can be analysed. It shows the reader the process of how a team or several teams can be used to discuss challenges and come up with a plan on how each of the scenarios can be analysed. It also shows how both quantitative and qualitative approaches should be used in conjunction with each other to give input from a wide perspective. The paper argues that a combination of the quantitative and the qualitative approaches is the best solution to achieve a representative selection of combined scenarios. The reason for this is given in the limited and narrow range of the quantitative method, and in the often subjective nature of the qualitative method.

\section{Acknowledgements}

The authors would like to thank the ten largest municipalities in Norway (VASK municipalities), the Norwegian interest group for water 'Norsk Vann' and the Norwegian Research Council for the financing and support of this work.

\section{Authors' contributions}

SB wrote the paper. SB conceived the overall study and its design. The sub case studies were conceived by both SB and SS. SS contributed to an overal feedback and input to the paper. The content of the paper is a product of discussions between SB and SS. Both authors read and approved the final manuscript.

\section{Competing interests}

The authors declare that they have no competing interests.

\section{Publisher's Note}

Springer Nature remains neutral with regard to jurisdictional claims in published maps and institutional affiliations.

Received: 8 September 2017 Accepted: 27 March 2018

Published online: 24 May 2018

\section{References}

1. Marlow DR, Moglia M, Cook S, Beale DJ (2013) Towards sustainable urban water management: a critical reassessment. Water Res 47(20):7150-7161

2. Postma TJBM, Liebl F (2005) How to improve scenario analysis as a strategic management tool? Tech Forecast Soc Chang 72(2):161-173

3. van Asselt, M. (2012) Foresight in action: developing policy-oriented scenarios. Published by Routledge, London

4. Rhisiart M, Miller R, Brooks S (2015) Learning to use the future: developing foresight capabilities through scenario processes. Tech Forecast Soc Chang 101:124-133

5. Waage, M. (2010) Nonstationarity water planning methods. In workshop on nonstationarity, hydrologic frequency analysis, and water management

6. Galloway, G.E. (2011) If stationarity is dead, What do we do now? Journal of the American Water Resources Association (JAWRA) 47(3):563-570.

7. Bood R, Postma T (1997) Strategic learning with scenarios. Eur Manag J 15(6):633-647

8. Makropoulos CK, Memon FA, Shirley-Smith C, Butler D (2008) Futures: an exploration of scenarios for sustainable urban water management. Water Policy 10(4):345-373

9. McCarl BA, Villavicencio X, Wu X (2008) Climate change and future analysis: is stationarity dying? Am J Agric Econ 90(5):1241-1247

10. Milly P, Betancourt J, Falkenmark M, Hirsch RM, Kundzewicz ZW, Lettenmaier DP, Stouffer RJ (2008) Stationarity is dead: whither water management? Science 319(5863):573-574

11. Brown RR, Farrelly MA (2009) Delivering sustainable urban water management: a review of the hurdles we face. Water Sci Technol 59(5):839-846
12. Marlow D, Beale D, Burn S (2010) A pathway to a more sustainable water sector: sustainability-based asset management. Water Sci Technol 61(5):1245

13. Ashley, R. and Tait, S. (2012) Use of scenarios in PREPARED, in deliverable D6.2.7. PREPARED project, funded by the 7th European Framework

14. van der Zouwen M, Segrave A, Büscher C, Monteiro A J, Galvão A, Ramoa A, Hochstrat R (2015) Guidelines for urban water strategic planning: inspiration from theories and best practices, in TRUST deliverable D12.1C

15. Blackmore J, Wang X, Wang C-H, Yum K-K, Diaper C, Zhou M, McGregor G (2008) Risk assessment and management: a guide for integrated urban water systems. eWater Technical Report. eWater Cooperative Research Centre, Canberra

16. Hallegatte S (2009) Strategies to adapt to an uncertain climate change. Glob Environ Chang 19(2):240-247

17. van't Klooster SA, van Asselt BA (2006) Practising the scenario-axes technique. Futures 38(1):15-30

18. Behzadian K, Morley M, Vitorino D, Coelho S, Ugarelli R, Kapelan Z (2015) Final report with DSS methodology, software and case study from a pilot city. Deliverable 54.3, in TRUST: TRansitions to the Urban Water Services of Tomorrow. European Union Seventh Framework Program

19. Miller KD, Waller HG (2003) Scenarios, real options and integrated risk management. Long Range Plan 36(1):93-107

20. Lempert R, Popper S, Bankes S (2002) Confronting surprise. Soc Sci Comput Rev 20(4):420-440

21. Bishop P, Hines A, Collins T (2007) The current state of scenario development: an overview of techniques. Foresight 9(1):5-25

22. Schoemaker PJ (1995) Scenario planning: a tool for strategic thinking. Sloan Manag Rev 36(2):25

23. Voros J (2003) A generic foresight process framework. Foresight 5(3):10-21

24. Herz RK (2002) Software for strategic network rehabilitation and investment planning. International conference. Computer Aided Rehabilitation of Water Networks CARE-W. Dresden, Germany, 65-84

25. Meissner $\mathrm{P}$, Wulf $\mathrm{T}$ (2013) Cognitive benefits of scenario planning: its impact on biases and decision quality. Technol Forecast Soc Chang 80(4):801-814

26. Duinker PN, Greig LA (2007) Scenario analysis in environmental impact assessment: improving explorations of the future. Environmen Impact Assess Rev 27(3):206-219

27. Varum CA, Melo C (2010) Directions in scenario planning literature-a review of the past decades. Futures 42(4):355-369

28. Amer M, Daim TU, Jetter A (2013) A review of scenario planning. Futures 46:23-40

29. Börjeson L, Höjer M, Dreborg K-H, Ekvall T, Finnveden G (2006) Scenario types and techniques: towards a user's guide. Futures 38(7):723-739

30. Sedlak DL (2014) 2014 Clarke Prize Lecture: Delivering the Fourth WaterRevolution. NWRI Clarke Prize Conference. Huntington Beach California

31. McDonald RI (2008) Global urbanization: can ecologists identify a sustainable way forward? Front Ecol Environ 6(2):99-104

32. Varis O, Somlyódy L (1997) Global urbanization and urban water: can sustainability be afforded? Water Sci Technol 35(9):21-32

33. Herz R, Lipkow A (2003) Strategic water network rehabilitation planning. Water Sci Technol Water Supply 3(1-2):35-42

34. ISO (2009) Risk management-principles and guidelines, in ISO 31000:2009. International Organization for Standardization, Geneva

35. Rausand M (2013) Risk assessment: theory, methods, and applications. Volume 115. Published by John Wiley \& Sons

36. Ugarelli R, Røstum J (2012) Risks and interdependencies in critical infrastructures—a guideline for analysis. In: Hokstad P, Bower UI, Vatn J (eds) Integrated urban water system. Springer, London

37. Ugarelli, R., Ceu Almeida, M., Behzadian, K., Liserra, T., Smeets, P., Kapelan, Z. and Sægrov, S. (2014) Sustainability risk based assessment of integrated urban water system. In 11th International Conference on Hydroinformatics, HIC

38. Hanssen-Bauer I, Førland EJ, Haddeland I, Hisdal H, Mayer S, Nesje A, Nilsen JEØ, Sandven S, Sandø AB, and Sorteberg A (2015) Klima i Norge 2100 Kunnskapsgrunnlag for klimatilpasning oppdatert i 2015, in NCCS report, NCCS. Miljødirektoratet, Oslo, 203

39. Ugarelli R, Leitão JP, do Céu Almeida M, and Bruaset S (2010) Overview of climate change effects which may impact the urban water cycle - D2.2.1, in European Union Seventh Framework Program. European Union

40. Simonsen $A B$ (2016) $A$ qualitative and quantitative assessment of the long-term hydraulic reliability of Norwegian water distribution network. Norwegian University of Science and Technology, Trondheim 